\title{
Leydig cell ovarian tumor - clinical case description and literature review
}

\author{
Mateusz Klimek ${ }^{1}$, Patrycja Radosz ${ }^{2}$, Magdalena Lemm ${ }^{1}$, Wojciech Szanecki ${ }^{1}$, Aleksandra Dudek², \\ Sandra Pokładek ${ }^{2}$, Maria Piwowarczyk ${ }^{2}$, Michał Poński ${ }^{1}$, Bartosz Cichońn ${ }^{1}$, Maciej Kajor ${ }^{3}$, Andrzej Witek $^{1}$ \\ ${ }^{1}$ Department of Gynaecology, Medical University of Silesia, Katowice, Poland \\ ${ }^{2}$ Students' Scientific Society, Department of Gynaecology, Medical University of Silesia, Katowice, Poland \\ ${ }^{3}$ Department of Pathomorphology, Medical University of Silesia, Katowice, Poland
}

\begin{abstract}
Leydig cell ovarian tumors constitute not only a medical problem for clinicians but also a social problem which is why women with symptoms of hirsutism relatively quickly contact physicians for medical consultation. Leydig cell ovarian tumor is a rare sex cord-gonadal stromal tumor which constitutes less than $0.5 \%$ of ovarian tumors. These cancers appear at all ages but the majority of the cases concern women in the perimenopause. In the majority of cases (70-85\%), the growth is accompanied by androgen secretion, together with virilization and hirsutism. The presence of hormonally active ovarian cancers should be suspected in cases of rapidly growing symptoms of masculinization, especially when the level of free testosterone in the blood exceeds the upper limit for the given age more than three times. In diagnosing postmenopausal hyperandrogenism, it is necessary to take into account hormonally active ovarian tumors, as well as adrenal cancers. It is important to exclude other causes of hyperandrogenism, e.g. endocrinopathies (acromegaly or hypothyroidism), or iatrogenic and idiopathic factors. In order to make the diagnosis and implement the proper treatment method faster, an interdisciplinary team of physicians specializing in endocrinology, gynecology and oncology is crucial. This paper contains a study of two cases concerning Leydig cell ovarian tumors in women of postmenopausal age with symptoms of masculinization and hirsutism.
\end{abstract}

Key words: hirsutism, menopause, androgens, ovary.

\section{Introduction}

In women, hyperandrogenism manifests itself through excessive body hair growth in the male pattern, balding in the male pattern, acne, together with menstrual disorders, a deeper voice and other features of virilization during the reproductive period. Around $25 \%$ of women in the reproductive period present features of hirsutism, which in most cases is caused by the polycystic ovary syndrome $[1,2]$. In less than $5 \%$ of the female patients with excessive male pattern body hair, testosterone secretion by ovarian or adrenal tumors is diagnosed [3]. Hormonal tests which in both cases indicate raised levels of testosterone and androstenedione are helpful in making an accurate diagnosis. DHEA-S occurs significantly more often in cases of adrenopathies.

Hirsutism can be caused by many factors. These can be divided into distinct familial phenotypes: ovarian and adrenal, other endocrinopathies or iatrogenic and idiopathic factors.

Taking an accurate and detailed medical history, conducting a physical examination of the patient to- gether with medical imaging, and biochemical tests of sex hormone levels play a significant role in differential diagnosis. A sudden onset of intense androgenization with rapidly growing symptoms of masculinization suggest a clinical diagnosis of a malignant ovarian or adrenal tumor.

Among ovarian tumors, hormonal activity is most often present in gonadal tissue neoplasms, which constitute about $5 \%$ of all ovarian tumors [4]. In cases of gonadal tissue neoplasms, one can sporadically observe cancers arising from Sertoli and Leydig cells. Androgen-secreting tumors account for $<1 \%$ of all ovarian tumors and most of them are benign [5]. The new classification system classifies these neoplasms as lipid cell tumors. They often resemble male gonads in their structure and hormonal activity. If hyperandrogenism is accompanied by a hyperestrogenic state, it is often caused by co-occurrence of endometrial cancer. Tumors composed only of Leydig cells are observed most often in the postmenopausal period [6].

Suspicion of tumor is an indication for conducting transvaginal ultrasonography in order to evaluate the ovaries, and computed tomography in order to ex- 
clude adrenopathies. Positron emission tomography with fluorodeoxyglucose as a marker can be used as an alternative method for detecting considerably small foci of Leydig cell ovarian tumors [9]. It is important to remember that the lack of features characteristic of virilization and masculinization does not exclude Leydig cell ovarian neoplasm. In $25-30 \%$ of cases, there is no increase in the level of androgens [10].

It is recommended to conduct diagnostic laparoscopy for cases in which the medical condition indicates virilization but the medical imaging does not show any standard deviation. Such a procedure is dictated by the small sizes of the hormonally active tumors. In $98 \%$ of the cases, ovarian tumors occur only on one side, being located either in the ovarian sinus or the ovarian medulla [7]. If the laparoscopy does not show any lesions in the ovaries and adrenal glands, it is recommended that blood samples be collected from both ovarian and suprarenal veins in order to determine the androgen level [8]. An increased level of testosterone in one of the samples indicates that there is a hormonally active neoplasm present in this structure.

$90 \%$ of the diagnosed Leydig cell ovarian tumor cases are classified as stage I cancer according to the FIGO staging system. This female patient group is characterized by a positive prognosis after operational treatment, and the survival rate is estimated at 84 $95 \%$ [11].

The gold standard for treatment of these cases is hysterectomy with removal of the adnexa with comprehensive staging. If a woman in her reproductive period wishes to maintain her fertility, one side adnexectomy is also taken into account. Patients with stage IA-IC desiring to preserve their fertility could be treated by fertility-sparing surgery and lymphadenectomy can be skipped. In older women or patients at an advanced stage, removal of the uterus, ovaries, the omentum, and (to the extent possible) metastatic deposits is considered to be standard surgery [12]. In advanced cases a follow-up treatment is recommended either in the BEP combination (bleomycin + etoposide + cisplatin), VAC combination (vinblastine + doxorubicin + cisplatin) or paclitaxel/carboplatin. If the tumor relapses, recurrent surgery, radiotherapy, chemotherapy, and potentially hormonotherapy is used [4]. Patient surveillance and patient follow-up are crucial after the surgical treatment.

In histopathologic examination, it is typical to see steroid cells filled with bright or eosinophilic cytoplasm with Reinke crystals which are protein inclusions and cytoplasmic lipofuscin inclusion. In the closest area to the lesions, the organ's architecture changes. Foci of stromatosis may appear. The mitotic index is usually low, which is reflected in the non-violent nature of the clinical course. Apart from the typical microscopic imaging, positive immunohistochemical staining of al- pha-inhibin and vimentin is important to establish the diagnosis. Positive reactions are also observed with cytokeratin CAM 5.2, cytokeratin AE1/AE3, actin, EMA and protein $\mathrm{S} 100$.

\section{Case reports}

Cose 1

A 61-year old female patient was electively admitted to the clinic with symptoms of hirsutism for further diagnosis and treatment of a virilizing tumor of the left ovary. Medical history was taken, and the patient was examined. The patient did not complain of any ailments when admitted. The patient reported rare pains around the abdomen, frequent urination, and a deeper voice lasting for two years. The gynecological history showed that the patient had her first menstrual period at the age of 13 , and the last at the age of 50 . The cycles were painless and regular. The patient gave birth twice naturally.

The patient was admitted in a good general condition. The hirsutism was evaluated at 18/36 on the Ferriman-Gallwey scale (Fig. 1A and 1B). The gynecological examination revealed: a multiparous vulva and vagina and an enlarged, thickened, and uneven uterus with leiomyoma lesions. The areas of the adnexa were free of any palpably detectable pathological lesions on both sides. On speculum the cervix had a normal view without any ectopy.

Additional laboratory tests and imaging examinations were ordered. Outpatient examination revealed an increased total testosterone level of $769 \mathrm{ng} / \mathrm{dl}$ (normal range: $2.9-40.8 \mathrm{ng} / \mathrm{dl}$ ) and increased dehydroepiandrosterone level of $225 \mu \mathrm{g} / \mathrm{dl}$ (normal range: 18.9$205 \mu \mathrm{g} / \mathrm{dl}$ ). The level of CA125 was found to be within the normal range $(7.2 \mathrm{U} / \mathrm{ml}$ with the range between 0 and $35 \mathrm{U} / \mathrm{ml}$ ). The transvaginal ultrasonography revealed: an anteflexed uterine body of $80 \times 70 \mathrm{~mm}$ with an irregular outline and a coarse echo pattern, and with leiomyoma lesions on its entirety; endometrium with thickness of $10 \mathrm{~mm}$ modelled by leiomyomata; solid lesion of $40 \times 35 \mathrm{~mm}$ with low vascularisation and smooth border found within the left ovary, ovary enlarged. The right ovary had a normal structure. Negligible fluid was found in the pouch of Douglas.

The patient was informed of the diagnosis, possible methods of treatment and the risks involved. She expressed informed consent for the treatment suggested. On the second day after admission, the patient underwent laparotomy. The operation revealed a left ovarian tumor with an uneven surface adhering to the omentum and intestines, and an enlarged uterus with intramural myoma up to $70 \mathrm{~mm}$ up to the cervix. It was necessary to separate the adhesions between the left ovary and the intestines, and between the ovaries and the oviducts. Due to the location of the myoma a hysterectomy 

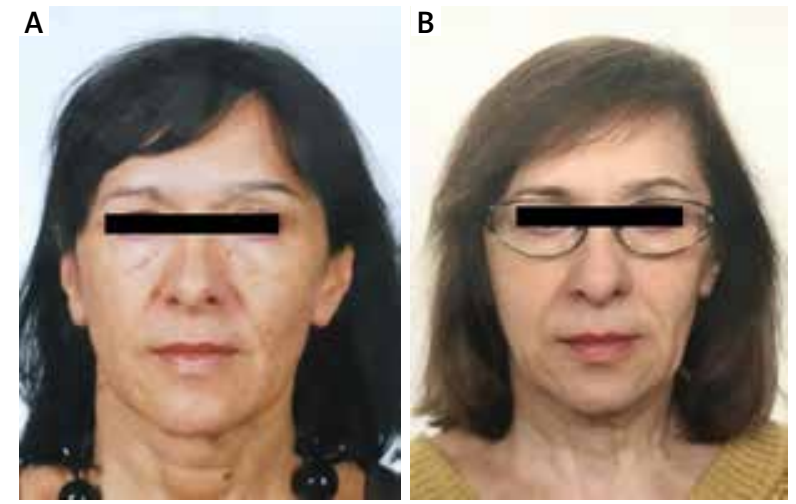

Fig. 1. A) Before the clinical manifestation of hyperandrogenism. B) Present excessive face hair removed by beauty treatments. Visible changes of facial shape and thinning of the hair

with removal of adnexa on both sides was performed. During the operation, appropriate samples were collected for histopathologic examination. The treatment was completed without significant complications. After the treatment, the patient was given anesthetics, and was subjected to fluid therapy and antibiotic therapy.

The histopathologic examination revealed a Leydig cell tumor with Reinke crystals (Fig. 2A). In the immunohistochemical examination, the tumor cells became positively stained for the presence of inhibin and negatively for cytokeratin in individual tumor cells (Fig. 2B and $2 \mathrm{C}$ ). The analyzed specimen indicated a benign tumor. There was no infiltration of blood vessels or nerves. The examination also revealed the presence of a leiomyoma/thecoma tumor in the vicinity of the right adnexa. An outpatient examination carried out around 2 months after the patient was discharged showed that the total testosterone levels in the blood became normalized to the level of $22.56 \mathrm{ng} / \mathrm{dl}$ (normal range: 2.9-40.8 ng/dl).

\section{Case 2}

A 60-year old female patient was electively admitted to the clinic with symptoms of virilization for further diagnosis and treatment of the hormonally active
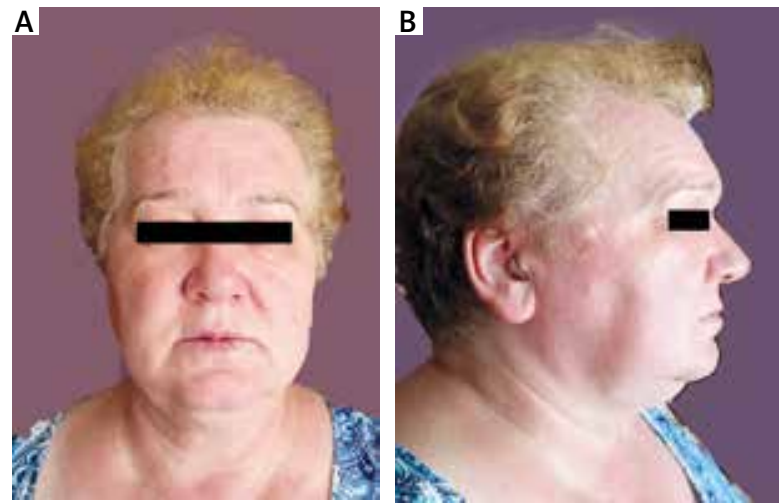

Fig. 3. Male type androgenic alopecia presented as hair loss in front of the scalp. Receding hairline and thinning of the hair

tumor of the left ovary. Medical history was taken, and the patient was examined. The patient did not complain of any ailments when admitted. The patient indicated that the first symptoms of excessive body hair appeared around 3 years before being admitted. This was accompanied by a gradually deeper voice, shrinking breast glands and male type of alopecia (Fig. $3 \mathrm{~A}$ and 3B). Around 2 years before admission, she started to lose hair around the temples. The gynecological history showed that the patient had her first menstrual period at the age of 14, and the last at the age of 57 . The monthly cycles were regular. The patient was pregnant three times, and gave birth twice naturally.

The patient was admitted in a good general condition, and in a cardiovascularly and respiratorily stable state. Physical examination revealed: obesity, excessive subcutaneous tissue and hirsutism at 15/36 on the Ferriman-Gallwey scale. The bimanual gynecological examination and speculum examination revealed: a multiparous vulva and vagina, a proper ectocervix, and a mobile anteflexed uterus; adnexa on both sides were not examined. The change described in the admission order was palpably not detectable during the examination.

Laboratory tests and imaging examinations were ordered. Particular attention was drawn to the raised
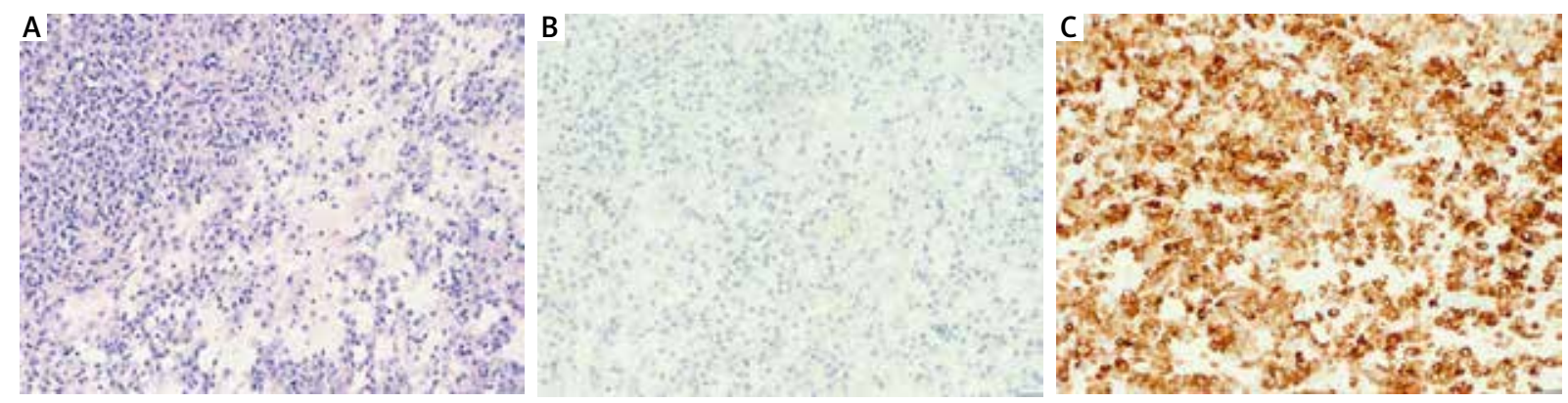

Fig. 2. A) E\&H, cells mimic the developmental phases of the nuclei by forming bands/coils ( $\times 100$ enlargement). B, C) An immunohistochemical study. Negatively stained cells with antibodies for cytokeratin (B) and for inhibin (C) positive $(\times 100-1000$ enlargement) 
levels of free testosterone, the concentration of which was $1.75 \mathrm{ng} / \mathrm{ml}$ (normal range: 0.029-0.408 ng/ml), and of DHEA-S, the concentration of which was 249.5 $\mu \mathrm{g} / \mathrm{dl}$, with the norm being 18.9-205.0 $\mu \mathrm{g} / \mathrm{dl}$. The CA125 marker was deemed not to be raised $(24.37 \mathrm{U} / \mathrm{ml}$, with the norm being 0-35 U/ml). The ultrasound conducted on the first day after admission revealed: an anteflexed uterine body with an irregular outline, a coarse echo pattern, and numerous Nabothian cysts. On the left side, the ovary behind the uterus featured a unilocular solid tumor with smooth border and low vascularisation, and with a diameter of $29 \times 37 \mathrm{~mm}$; endometrium with thickness of $8 \mathrm{~mm}$. No adnexa on the right side were revealed. No free fluids in the pouch of Douglas were detected.

The patient was informed of the diagnosis made, possible methods of treatment and the risks involved. The patient expressed informed consent for the treatment suggested. On the second day after admission, the patient underwent laparotomy with supracervical removal of the uterine body and both ovaries. The operation revealed the unlarged left ovary with a solid tumor up to $50 \mathrm{~mm}$, the right ovary without any pathology and normal uterus. Appropriate samples were collected for histopathologic examination. The treatment did not encounter any significant complications.

The histopathologic examination revealed typical view of a benign Leydig cell ovarian tumor with Reinke crystals. There were no infiltrated blood vessels or nerves. In the immunohistochemical examination, the tumor cells stained positively for the presence of inhibin.

\section{Conclusions}

Leydig cell ovarian tumors constitute not only a medical problem for clinicians but also a social problem - which is why women with symptoms of hirsutism relatively quickly contact physicians for medical consultation. Thanks to this, $90 \%$ of the cases are detected at stage I of neoplasm progression according to the FIGO staging system. By default, the patients undergo adnexectomy or radical hysterectomy.

In order to establish the diagnosis and implement the proper treatment method faster, an interdisciplinary team of physicians specializing in endocrinology, gynecology and oncology is crucial. In diagnosing postmenopausal hyperandrogenism, it is necessary to take into account hormonally active ovarian tumors, as well as adrenal cancers. Furthermore, imaging results for the abdomen and lesser pelvis are also highly significant.

Leydig cell tumors are uncommon neoplasms arising from gonadal stroma. Most cases of postmenopausal virilizing ovarian cancers are benign, and bilateral salpingo-oophorectomy is an appropriate cure. In the absence of any sign of histopathological or surgical ma- lignancy, long-term follow-up is necessary to exclude recurrence or metastasis.

\section{Disclosure}

The author reports no conflict of interest.

\section{References}

1. Escobar-Morreale HF, Carmina E, Dewailly D, et al. Epidemiology, diagnosis and management of hirsutism: A consensus statement by the androgen excess and polycystic ovary syndrome society. Hum Reprod Update 2012; 18: 146-170.

2. Glintborg D, Andersen M. An update on the pathogenesis, inflammation, and metabolism in hirsutism and polycystic ovary syndrome. Gynecol Endocrinol 2010; 26: 281-296.

3. Glintborg D, Altinok ML, Petersen KR, Ravn P. Total testosterone levels are often more than three times elevated in patients with androgensecreting tumours. BMJ Case Rep 2015; 2015.

4. Reed N, Millan D, Verheijen R, Castiglione M. Non-epithelial ovarian cancer: ESMO clinical practice guidelines for diagnosis, treatment and follow-up. Ann Oncol 2010; 21 (Suppl 5): v31-36.

5. Ajith S, Beena G, Mathew N, Omana E. Postmenopausal hyperandrogenism of ovarian origin: A clinicopathologic study of five cases. J Midlife Health 2016; 7: 189-192.

6. Chen M, Zhou W, Zhang Z, et al. An ovarian Leydig cell tumor of ultrasound negative in a postmenopausal woman with hirsutism and hyperandrogenism: A case report. Medicine (Baltimore) 2018; 97: 8-11.

7. Shanbhogue AKP, Shanbhogue DKP, Prasad SR, et al. Clinical syndromes associated with ovarian neoplasms: A comprehensive review. Radiographics 2010; 30: 903-919.

8. Kaltsas GA, Mukherjee JJ, Kola B, et al. Is ovarian and adrenal venous catheterization and sampling helpful in the investigation of hyperandrogenic women? Clin Endocrinol (Oxf) 2003; 59: 34-43.

9. Prassopoulos V, Laspas F, Vlachou F, et al. Leydig cell tumour of the ovary localised with Positron Emission Tomography/Computed Tomography. Gynecol Endocrinol 2011; 27: 837-839.

10. Verdorfer I, Horst D, Höllrigl A, et al. Sertoli-Leydig cell tumours of the ovary and testis: A CGH and FISH study. Virchows Arch 2007; 450: 267271.

11. Markowska J, Mardas M. Sex cord stromal tumors of the ovary: principles of management. Curr Gynecol Oncol 2010; 8: 265-272.

12. Ciebiera M, Baran A, Słabuszewska-Jóźwiak A, et al. Sertoli-Leydig cell tumour (SLCT) - the case of a $15 \mathrm{~cm}$ diameter ovarian tumour with negative markers and absent hormonal symptoms. J Health Inequal 2019; 5: 117-119. 